\title{
ARTICLE
}

\section{The transbilayer distribution of polyunsaturated phospholipids determines their facilitating effect on membrane deformation}

Received 00th January 20xx Accepted 00th January 20xx DOI: $10.1039 / \times 0 \times x 00000 x$

\begin{abstract}
Marion Tiberti ${ }^{\mathrm{a}}$ Bruno Antonny ${ }^{* \mathrm{a}}$ and Romain Gautier ${ }^{\mathrm{a}}$
In the cell, membrane deformation and fission (collectively refered as to 'budding') is driven by specific protein machineries but is also influenced by lipid composition. We previously reported that phospholipids with polyunsaturated acyl chains facilitate membrane budding because they adapt their shape to membrane curvature, thereby decreasing membrane bending rigidity. The facilitating effect of polyunsaturated lipids was observed in experiments and simulations performed on membranes where the two bilayer leaflets had the same lipid composition. However, biological membranes are generally asymmetric. Here, we present coarse-grained molecular dynamics simulations on asymmetric phospholipid bilayers undergoing deformation via a pulling force along the bilayer normal. One leaflet contains monounsaturated C18:0-C18:1-phospholipids, whereas the opposite leaflet contains polyunsaturated C18:0-C22:6-phospholipids. When present in the monolayer orientated towards the pulling force and thereby in the convex face of the forming tube, C18:0C22:6-phospholipids facilitate membrane tubulation. In contrast, C18:0-C22:6-phospholipids in the concave face of the tube have no effect. Analysis of lipid shape indicates that these contrasting effects arise from the superior ability of polyunsaturated phospholipids to swell in the convex leaflet, whereas mono and polyunsaturated phospholipids behave similarly in the concave leaflet. The leaflet-dependent effect of polyunsaturated phospholipids matches well their asymmetric distribution in biological membranes, notably in synaptic vesicles, which are produced by the fastest budding event

body.
\end{abstract}

\section{Introduction}

Formation of transport vesicles on membrane-bound organelles and at the plasma membrane is driven by the assembly of protein coats, which impose a spherical shape to the membrane, and by proteins acting at the bud neck to promote membrane fission ${ }^{1}$. In addition to these specific machineries, other factors contribute to the efficiency of vesicle formation. These include cytoskeleton proteins, molecular motors, and intrinsic physical membrane properties such as protein crowding, membrane tension or membrane bending rigidity ${ }^{2}$.

Rigidity, which describes the resistance of the membrane to bending, depends on the lipid composition ${ }^{3}$. For example, saturated lipids, which increase membrane rigidity, reduce the efficiency of protein coat-mediated membrane budding ${ }^{4}$, whereas polyunsaturated phospholipids, which reduce membrane rigidity, facilitate membrane deformation and fission $^{3,5,6}$. The effects of lipid unsaturation on membrane budding might be physiologically relevant since the acyl chain

\footnotetext{
a. Université Côte d'Azur et CNRS, IPMC, 660 route des lucioles 06560 Valbonne France

*Corresponding authors: antonny@ipmc.cnrs.fr; qautier@ipmc.cnrs.fr

† Footnotes relating to the title and/or authors should appear here.

Electronic Supplementary Information (ESI) available: [details of any supplementary information available should be included here]. See DOI: $10.1039 / x 0 x \times 00000 x$
}

profiles of phospholipids shows striking differences between cell types and between organelles ${ }^{7,8}$. Notably, synaptic vesicles, which are the smallest transport vesicles and form by an ultrafast endocytic event, are extremely rich in phospholipids with a polyunsaturated acyl chain (C20:4 or C22:6) at position sn2 of the glycerol backbone ${ }^{9,10}$. Furthermore, manipulating the acyl chain profile of the membranes of model cell lines affects endocytosis ${ }^{6,11}$.

Numerous experimental approaches have been developed to study membrane budding events, from cellular observations to reconstitutions with well-defined lipid and protein components $^{12}$. In addition, coarse-grained molecular dynamics simulations have been designed to mimic membrane deformation ${ }^{13}$.

By combining experiments and simulations, we previously addressed the impact of phospholipid polyunsaturation on membrane budding ${ }^{5,6}$. Membrane enrichment in C22:6containing phospholipids dramatically increases the budding efficiency of the endocytic proteins endophilin and dynamin both in cells and in vitro. In addition, a comprehensive analysis coupled to molecular dynamics simulations indicates that the efficiency of membrane deformation increases by two orders of magnitude depending on the number of polyunsaturated acyl chains carried by the phospholipids $(0<1<2)$ and the degree of acyl chain unsaturation (series 14:0 $<18: 1<18: 2<$ 
$18: 3<20: 4<22: 6$ ) . This large range results from the combination of two effects: a decrease in membrane rigidity per se, but also an increase in membrane permeability. Overall, lipids with one saturated acyl chain and one polyunsaturated acyl chain (e.g.

18:0-22:6) provide the best compromise for keeping the permeability of the membrane at low levels, while facilitating membrane budding 5 .

Our previous in vitro reconstitutions and simulations were performed with symmetric lipid bilayers. However, biological membranes are asymmetric. Historically, the transbilayer distribution of the main lipid classes was studied on the plasma membrane of erythrocyte cells ${ }^{14}$. Phosphatidylserine (PS) and phosphatidylethanolamine (PE) are mainly found in the inner (cytosolic) leaflet, whereas sphingomyelin (SM) and phosphatidylcholine (PC) are mostly present in the outer leaflet $^{15}$. This asymmetry in lipid classes should be accompanied by a strong asymmetry in the acyl chains. Indeed, PS and PE are the most polyunsaturated lipid classes in biological membranes. For example, PS and PE species containing 4 to 6 double bonds account for about 60 to $80 \mathrm{~mol}$ $\%$ of these phospholipids in the brain including synaptic vesicles ${ }^{10}$. In contrast, polyunsaturated PC species are less abundant ( $\approx 15 \mathrm{~mol} \%$ ) and SM is mostly saturated. Thus, the asymmetry of the plasma membrane in term of lipid classes should translate into a strong asymmetry in acyl chain content. This possibility was recently addressed by the Levental group who performed a comprehensive lipidomics analysis of the erythrocyte membrane ${ }^{16}$. Phospholipids with more than two unsaturations are much more abundant in the cytosolic leaflet than in the outer leaflet, notably due to the exclusive distribution of polyunsaturated PS and PE species such as 16:020:4 PS and 16:0-22:6 PE in the cytosolic leaflet. Differences in the diffusion rate of model peripheral proteins as well in the fluorescence properties of probes, which were added externally or internally, indicate that the cytosolic leaflet of plasma membrane of several cell types is more fluid than the outer leaflet ${ }^{16}$. Thus, the compositional differences between the two leaflets of the plasma membrane might contribute to distinct biophysical properties. However, whether the transbilayer distribution of polyunsaturated lipids has an influence on membrane bending rigidity has not been tested.

In this study, we used molecular dynamics simulations to examine whether the transbilayer distribution of polyunsaturated phospholipids in model membranes impacts membrane mechanics. We observed that polyunsaturated lipids facilitate membrane deformation when they are present in the convex leaflet (positive curvature) but not in the concave leaflet (negative curvature). This topology makes the plasma membrane of neurons well adapted to fast endocytosis.

\section{Computational Methods}

\section{Molecular dynamics simulations}

Our simulations were performed according to a previously described procedure ${ }^{13}$ that we adapted to membranes with a specific acyl chain profile ${ }^{5,6}$. Specifically, we used Martini coarse-grained lipid models (Wassenaar et al., 2015) to approximate monounsaturated 18:0-18:1 and polyunsaturated phospholipids 18:0-22:6 (Fig. 1A). The systems, which contained

$\approx 18000$ lipids solvated with a $100 \mathrm{~nm}$-thick water layer, were built by the Charmm-Gui tool adapted to coarse-grained

simulations and were equilibrated with a standard six-step process $^{17}$.

To simulate membrane deformation, we applied a force perpendicular to the initially flat bilayer (Fig. 1B). The force varied from 150 to $225 \mathrm{~kJ} \mathrm{~mol}^{-1} \mathrm{~nm}^{-1}$ and was applied to a lipid patch of $3 \mathrm{~nm}$ in radius (Fig. 1B). These lipids were restrained in the lateral plane $(x, y)$. Pulling simulations were performed with GROMACS $4.5^{17}$. The different membranes were simulated under a semi-isotropic condition and the periodic boundaries were applied in all directions. Lipids and water/ions were coupled separately. The time step was fixed at $20 \mathrm{fs}$ and the cut-off for the Lennard-Jones and electrostatic interactions was set at $1.2 \mathrm{~nm}$. The smooth particle-mesh was used to evaluate the electrostatic interactions. During the production runs, the $\mathrm{V}$-rescale thermostat and Berendsen barostat ${ }^{18}$ stabilized the temperature at $310 \mathrm{~K}$ and pressure at 1 bar, respectively. The simulations were performed for 200 ns or 400ns and were repeated three to five times for all conditions.

\section{Membrane composition}

All simulations with bilayers displaying symmetric lipid composition have been published and were used here for comparison $^{5}$.

To study the impact of the distribution of the polyunsaturated phospholipids between the two membrane leaflets, we prepared PC bilayers in which one leaflet contained $0,25,50$, 75 or $100 \mathrm{~mol} \%$ of 18:0-22:6 PC whereas all other lipids were 18:0-18:1 PC. The resulting asymmetric system required slight adjustment of the total lipid number in each leaflet to maintain the area of the two leaflets equal.

To evaluate the impact of the polar head, we prepared asymmetric bilayers in which one leaflet was made solely of 18:0-18:1 PC, whereas the opposite leaflet contained 50 mol\% 18:0-18:1 PC and 50 mol\% of another phospholipid (PE or PS) with a defined acyl chain profile (either 18:0-18:1 or 18:022:6).

To better approach the composition of biological membranes, we built a multi-component system in which one leaflet consisted of 18:0-18:1 PC whereas the opposite leaflet mimicked the phospholipids of the neuronal plasma membrane both in term of polar head and acyl chain profile ${ }^{19}$. This leaflet is described in Table 1. As a control, we built a similar bilayer in which all polyunsaturated phospholipids were 
replaced by 18:0-18:1 phospholipids (Table 1). We used counter ions to neutralize all systems containing anionic lipid.

\section{Simulation analysis}

For each tube, we measured the following geometrical parameters. We estimated the length of the tube (including the base) by calculating the difference between $z_{\max }$ and $z_{\min }$ of the bilayer and subtracting membrane thickness. We calculated the elongation velocity from the slope between $t=20 \mathrm{~ns}$ and $t=100 \mathrm{~ns}$ of the tube kinetics. We estimated the tube radius $(r)$ for tubes
A $18: 0-18: 1 \mathrm{PC}$

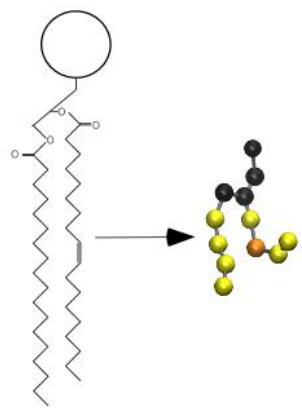

B
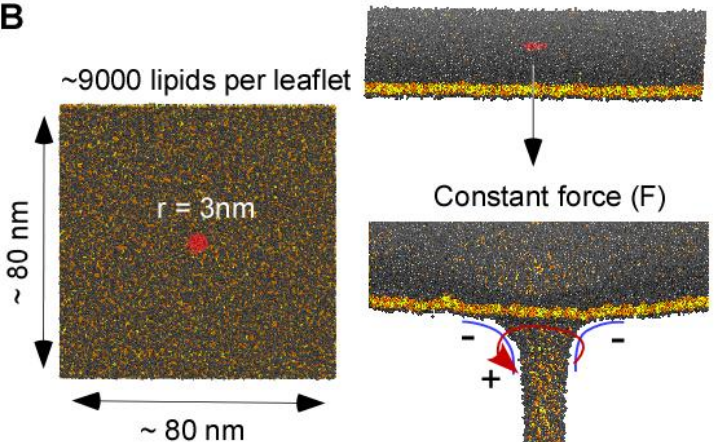

Constant force $(F)$

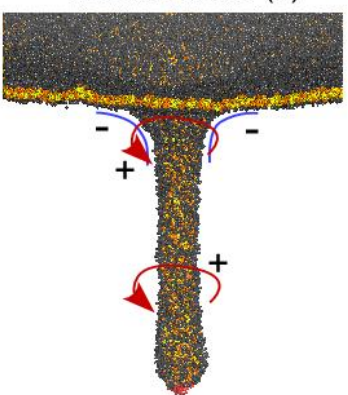

Fig 1 Principle of the molecular dynamics simulations. A. Phospholipid coarse-grained models. Black beads are polar head, yellow beads are saturated carbon and orange beads are unsaturated carbon. B. The pulling system. The phospholipids shown in red are the central patch from which we applied a constant pulling force, perpendicular to the initially flat membrane (left) to induce deformations leading different curvature of membrane (right): positive curvature in red and negative curvature in blue.

longer than $40 \mathrm{~nm}$. For this, we divided each tube into $4 \mathrm{~nm}$ slices and the radius of each slide was obtained by averaging the distance between the center of the tube and the nitrogen beads (NC3 in PC, NH3 in PE and CNO in PS) that compose the outer leaflet (outer radius) or the inner leaflet (inner radius). The final radius $(r)$ was the average of the radius of all slices. The tube curvature (c2) was given by $1 / r$.

To obtain the curvature at the base of the tube (c1), we determined the radius of an osculating circle.

The area per lipid ( $A P L)$ was calculated from the lateral surface of the entire tube by the following formula:

\section{$A P L=2 \pi r h / n$}

where $h$ is the estimated height of tube (without the base and dome), $r$ is the radius and $n$ is the number of lipids in this region.

For the conformation analysis, we used two methods. One to provide information on the conformation of the sn2 acyl chains. The second one provided information on the overall phospholipid geometry.

The bending angle of sn2 acyl chains was calculated between GL1-D2A-CA4 and between D1A-D3A-D5A for monounsaturated and polyunsaturated phospholipids, respectively. To cluster the acyl chain conformations, we divided them into 3 groups: straight $\left(>120^{\circ}\right)$, moderately kinked (between $70^{\circ}$ and $120^{\circ}$ ), and highly kinked $\left(<70^{\circ}\right)$.

To assess the effect of membrane curvature on phospholipid geometry, we overlapped all lipids of the tube that are in the same leaflet (excluding the base and the dome). To do this, we used the lipid cylindrical coordinates. By rotation and translation operations, all lipids were aligned on their GL1 bead without modifying their intrinsic geometry. Thereafter, we projected the lipids onto a grid ( $75 \times 75$ squares) and used a grey color logarithmic scale to represent the number of beads in each square. The resulting overlays were represented in two ways; one in which we separately analyzed the sn1 and sn2 chains; one in which we considered all beads from the two acyl chains.

Table 1 : Composition of the outer leaflet of the multicomponent system. Top: neuronal plasma membrane; Bottom: control. PI: phosphatidylinositol.

\begin{tabular}{|c|c|c|c|c|}
\hline & $\mathrm{PC}(27.3 \%)$ & $\begin{array}{l}\text { PE } \\
(41.8 \%)\end{array}$ & PS (20\%) & PI (10.2\%) \\
\hline $18: 0-18: 0$ & $23.1 \%$ & - & $4.6 \%$ & - \\
\hline 18:0-18:1 & $37.2 \%$ & $12.1 \%$ & $19.5 \%$ & $27.9 \%$ \\
\hline 18:1-18:1 & $10 \%$ & - & $21.8 \%$ & - \\
\hline $18: 0-20: 4$ & $20.2 \%$ & $35.2 \%$ & $21.8 \%$ & $27.9 \%$ \\
\hline \multirow[t]{2}{*}{$18: 0-22: 6$} & $9.5 \%$ & $52.7 \%$ & $23.2 \%$ & $44.1 \%$ \\
\hline & PC (27.9\%) & PE (41.6\%) & PS(20.5\%) & $\mathrm{PI}(10.2 \%)$ \\
\hline 18:0-18:0 & $23.5 \%$ & - & $4 \%$ & - \\
\hline 18:0-18:1 & $66.9 \%$ & $100 \%$ & $74 \%$ & $100 \%$ \\
\hline 18:1-18:1 & $9.6 \%$ & - & $22 \%$ & - \\
\hline
\end{tabular}

\section{Results}

3.1 The transbilayer distribution of polyunsaturated phospholipids determines their effect on membrane deformation

In previous simulations performed with symmetric PC membranes, we reported a strong facilitating effect of polyunsaturated lipids on membrane deformation ${ }^{5,6}$. Specifically, the rate of tube elongation (induced by a constant perpendicular force), was 3-4 times higher with 18:0-22:6 PC membranes compared to 18:0-18:1 PC membranes. To determine if this effect depends on the transbilayer distribution of polyunsaturated lipids, we performed similar 
simulations with asymmetric membranes. One leaflet contained monounsaturated 18:0-18:1 PC, whereas the other leaflet contained polyunsaturated 18:0-22:6 PC. We applied a constant force on one side or the other (Fig. 2A). Therefore, the polyunsaturated lipids were either in the outer (Fig. 2B left) or inner (Fig. 2B right) leaflet of the tube.

The snapshots of Fig. 2B and the quantifications of Fig. 2C-D show that the transbilayer distribution of polyunsaturated phospholipids had a dramatic effect on membrane
A I) 18:0-22:6 PC 18:0-18:1 PC symmetric membranes
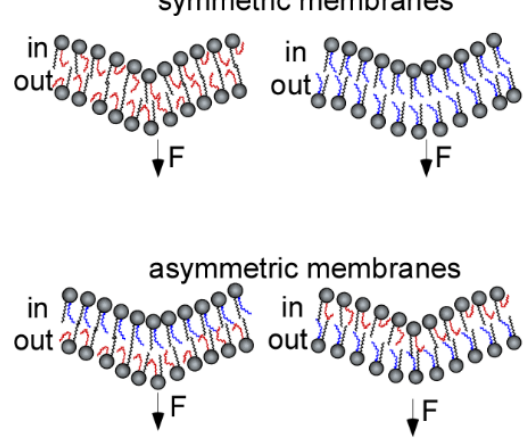

E

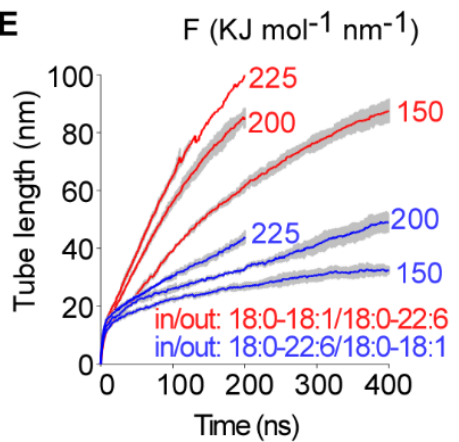

B
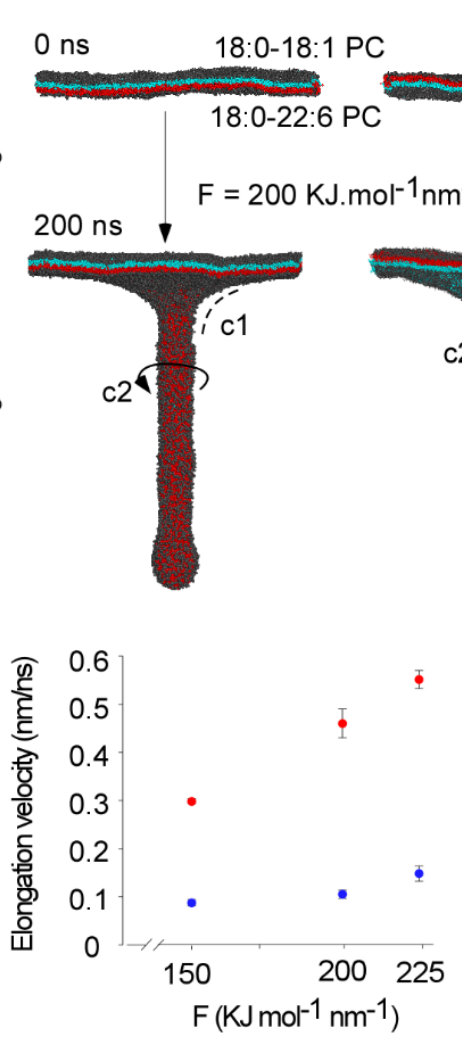

C

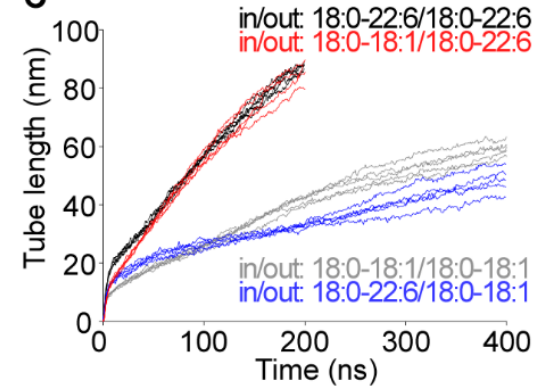

Fig 2 The transbilayer distribution of polyunsaturated PC determines its facilitating effect on membrane deformation. A. Schemes of the membrane models composed of 18:0-18:1 (blue) and/or 18:0-22:6 (red). B. Snapshots of membrane deformation at 0 or $200 \mathrm{~ns}$. C. Time course of tube pulling from symmetric 18:0-22:6 (black) or 18:0-18:1 (grey) PC membranes and from asymmetric PC membranes with 18:0-22:6 PC outside (red) or inside the tube (blue). D. Outer and inner tube radius for each membrane system and each replicate of pulling simulations. E. Left: time course of tube pulling from asymmetric membrane under increasing pulling forces. Right: Tube elongation velocity as a function of pulling force. F. Curvature values at the base and at the middle of the tube for each asymmetric membrane system (see panel $A$ for the definition of $c 1$ and $\mathrm{c} 2$ ). Errors bars in $D$ show standard deviation of the tube radius as measured from 80-100 snapshots of the same simulation over time. Error bars in $\mathrm{E}$ and $\mathrm{F}$ show standard deviation from three simulations $(E, F)$

deformation. When 18:0-22:6 PC was present in the outer leaflet of the tube, the overall process of elongation was fast and similar to that observed with pure symmetric 18:0-22:6 PC membranes (Fig. 2C). When 18:0-22:6 PC was present in the inner leaflet of the tube, elongation was slow and comparable to that observed with pure symmetric 18:0-18:1 PC membranes (Fig. 2C). Tube radius analysis showed that tubes with 18:0-22:6 PC in the outer leaflet and 18:0-18:1 PC in the inner leaflet were as thin as pure 18:0-22:6 PC tubes. In contrast, tubes with 18:0-18:1 PC in the outer leaflet and 18:022:6 PC in the inner leaflet had a larger diameter, similar to that observed with pure 18:0-18:1 PC tubes (Fig. 2D).

We repeated the tube extrusion protocol at varying forces, from 150 to $225 \mathrm{~kJ} \mathrm{~mol}^{-1} \mathrm{~nm}^{-1}$ (Fig. 2E). The elongation velocity increased with the applied vertical force and was always much faster when polyunsaturated lipids were present in the outer leaflet of the tube compared to the opposite distribution. In addition, membrane curvature at both the base and along the tube (Fig. 2B) was greater when 18:0-22:6 PC was on the outer leaflet of the tube (Fig. 2F).

To check that these results were not biased by the limiting membrane surface or the pulling area, we performed simulations under other conditions. First, we doubled the surface of the membrane.

Second, we enlarged both the membrane surface and the pulling area by 4-fold. As shown in Fig. S1, 18:0-22:6 PC facilitated membrane deformation in these systems only if present in the outer leaflet of the tube or the bud.

Altogether, these simulations indicate that it is not necessary to have polyunsaturated lipids on both leaflets to facilitate membrane deformation: polyunsaturated phospholipids are 
beneficial solely when present in the outside, i.e. convex, leaflet of the tube.

\subsection{Dose-dependent effect of 18:0-22:6 PC}

The concentration of polyunsaturated phospholipids in biological membranes varies from a few $\mathrm{mol} \%$ to up to $80 \mathrm{~mol}$ $\%$ depending on the organism, the tissue or the organelle $e^{7-9,20}$. We performed simulations in which we increased the amount of 18:0-22:6 PC from 0 to $100 \%$ in the outer or in the inner

A Force $200 \mathrm{KJ}_{\mathrm{mol}}{ }^{-1} \mathrm{~nm}^{-1}$

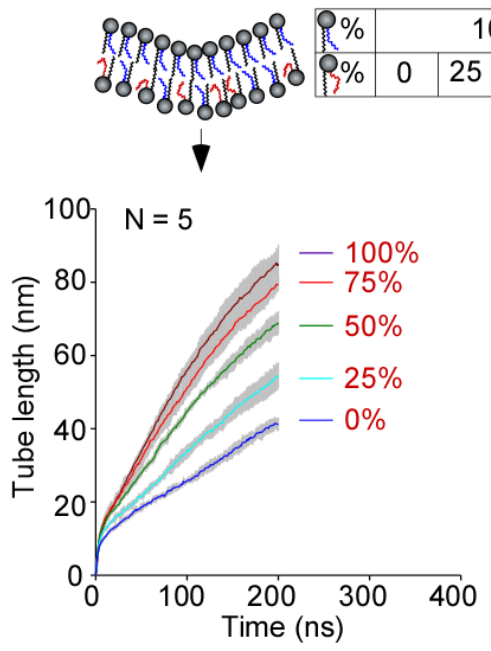

100

\begin{tabular}{|l|l|l|}
50 & 75 & 100 \\
\hline
\end{tabular}

B
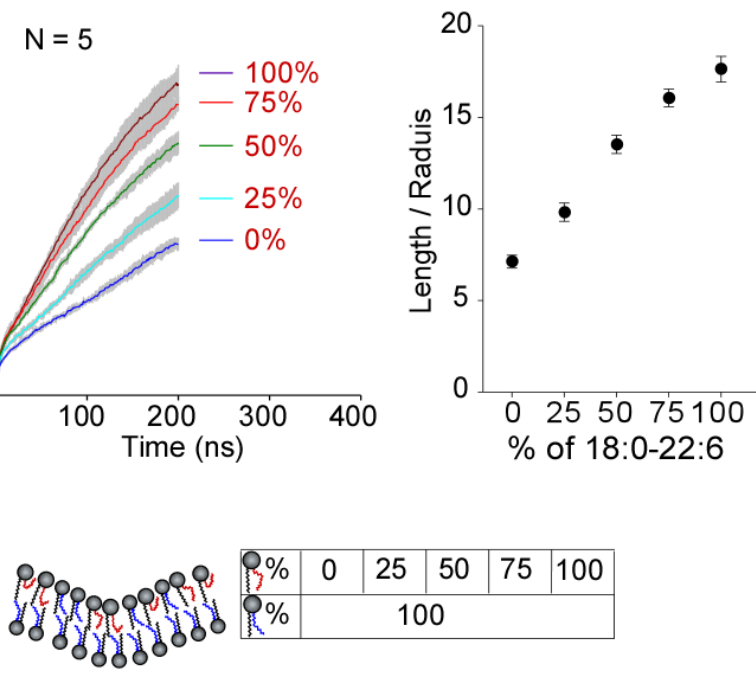

$\nabla$
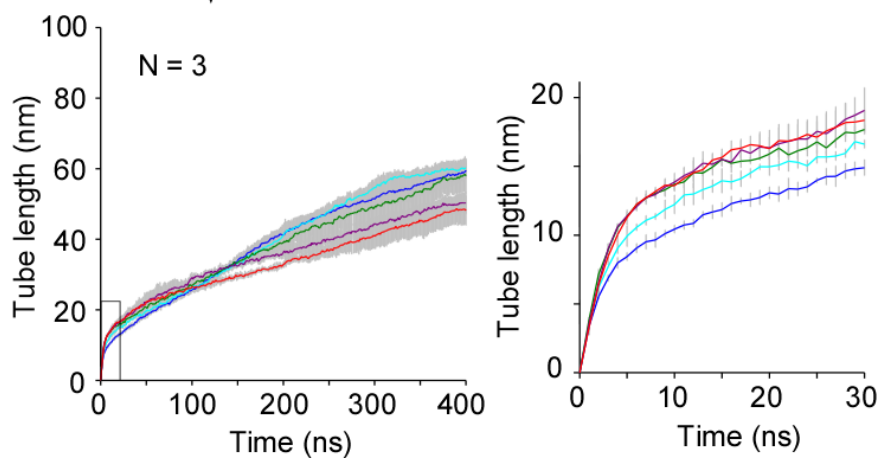

Fig 3 Dose response of the effect of 18:0 22:6 PC when present in the outer (A) or in the the inner (B) leaflet of the tube. The left panels show the times course of tube pulling as averaged from 3 or 5 replicas; the shaded area shows the SD. The right panel in A shows length/radius ratio of the tube at 200 ns. The right panel in B shows a zoom of the deformation kinetics at early stage.

leaflet of the tube. We observed a monotonous increase in the deformability of the membrane, both in length and in radius, when the fraction of 18:0-22:6 PC increased in the outer leaflet of the tube (Fig. 3A). In contrast, the presence of polyunsaturated phospholipids inside the tube was not beneficial for tube elongation (Fig. 3B). In the latter case, we noticed that the curves of tube elongation crossed (Fig. $2 \mathrm{C}$ and
3B). For the first $100 \mathrm{~ns}$, when a dome but not a full tube had just formed, the presence of 18:0-22:6 PC in the leaflet opposite to the pulling force facilitated membrane deformation. Thereafter, that is during tube elongation, the

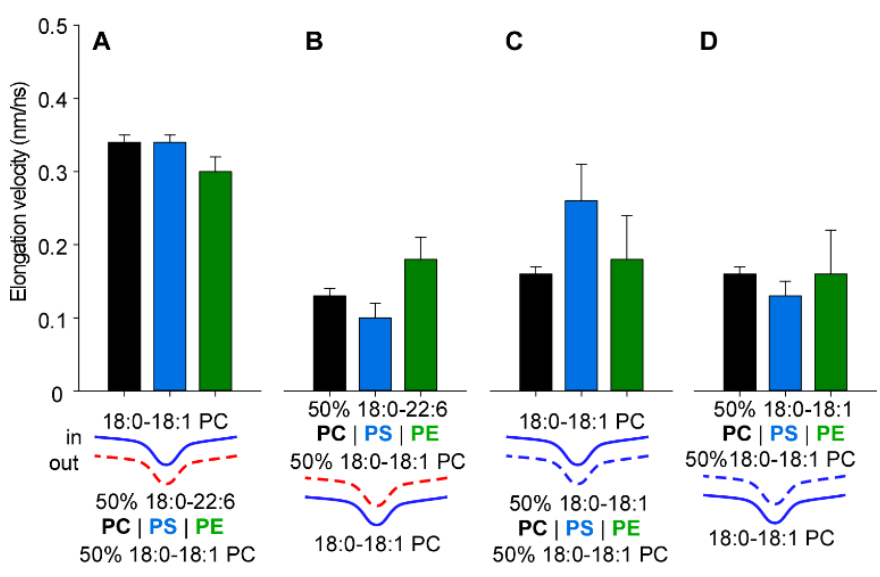

Fig 4 Effect of the lipid polar head group. Tube pulling was performed as in Figure 2B with bilayers in which $50 \%$ of PC in one leaflet was replaced by PE or PS. A, B. With 50\% 18:0-22:6 PC, PS or PE in the outer (A) or inner (B) leaflet of the deformation. C, D. With 50\% 18:0-18:1 PC, PS or PE in the outer (C) or inner (D) leaflet of the deformation. Error bars: standard deviations from three replica

presence of 18:0-22:6 PC was slightly inhibitory.

\subsection{Influence of the lipid polar head}

To determine if the effect of the transbilayer distribution of polyunsaturated lipids depends on the nature of the polar head, we next moved to more complex bilayers. The first system consisted in bilayers in which one leaflet contained 100 \% 18:0-18:1 PC whereas the other leaflet contained 50\% 18:018:1 PC and $50 \%$ PS or PE with a defined acyl chain profile (18:0-18:1 or 18:0-22:6).

In all cases, we observed that the chosen polyunsaturated phospholipid (PC, PE or PS) in the outer tube leaflet favored membrane deformation, whereas the opposite distribution did not (compare panels A and B in Fig. 4). In addition, the polar head had a slight impact. There was less difference in tube velocity when we inverted the inner/outer distribution of 18:022:6 PE as compared to what we observed with 18:0-22:6 PC or 18:0-22:6 PS. Owing to its small polar, PE might be more adapted to the inner leaflet of the tube than PC or PS.

We also evaluated the impact of the polar head in the context of pure monounsaturated phospholipids (Fig. 4C and D). Under these conditions, the elongation rates were very similar, except when 18:0-18:1 PS was present in the outer leaflet of the tube. Under this condition, membrane deformation was accelerated. The negative charge of the polar head might promote repulsion between neighboring lipids in the outer leaflet, thereby facilitating the growth of tube as experimentally observed ${ }^{21}$.

We concluded that the intrinsic impact of lipid polyunsaturation on membrane deformation weakly depends 


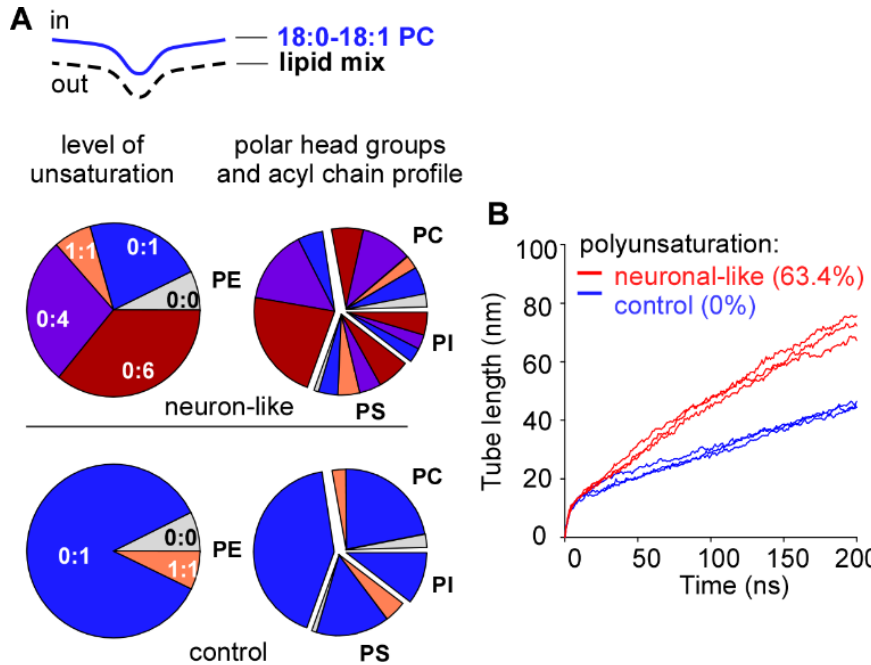

on the nature of the polar head. Moreover, this facilitating effect of 18:0-22:6 on membrane deformation is greater than those promoted by other physicochemical properties, such as size and charge of the polar head.

\subsection{Effect of polyunsaturated phospholipids in a model neuronal plasma membrane}

Fig 3 Tube elongation velocity from a bilayer with one leaflet mimicking the composition of the cytosolic leaflet of the neuronal plasma membrane. A. Scheme of the lipid composition. The inner leaflet of the tube contained 100\% 16:0-18:1 PC. The outer leaflet contained a fixed ratio of PC, PE, PS and PI with a defined acyl chain profile. The acyl chains were either highly polyunsaturated as found in neurons (top), or saturated/monosaturated (bottom). B. Time course of tube pulling from the bilaye described in $A$. Force $=200 \mathrm{~kJ} \mathrm{~mol}^{-1} \mathrm{~nm}^{-1}$. For each composition, three independent simulations are shown.

We next moved to the composition of the neuronal plasma membrane as previously used by the Marrink group in simulations $^{19}$. We focused only on the cytosolic leaflet, which, during endocytosis, becomes the outer leaflet of the tube generated in our simulations. The phospholipid composition of the cytosolic leaflet, which showed a high level of polyunsaturation (64 mol\%), is given in Table 1. For simplicity, the opposite leaflet was made of 18:0-18:1 PC. To evaluate the importance of lipid polyunsaturation, we prepared a control system, in which we replaced all polyunsaturated acyl chains (20:4 and 22:6) by monounsaturated (18:1) ones (Fig. 5A). Again, lipid polyunsaturation in the outer tube leaflet strongly favored elongation (Fig. 5B), suggesting that our results could be extended to more realistic membranes.

\subsection{Polyunsaturated phospholipids show conformational plasticity in the convex leaflet}

We suspected that the key role of the transbilayer distribution of polyunsaturated lipids on membrane deformation, might arise from a better adaptation of these lipids to positive curvature than to negative curvature. To address this hypothesis, we performed a molecular analysis by computing various parameters, including the area per lipid, the acyl chain flexibility, and the lipid shape.
The area per lipid, which corresponds to the ratio between the number of lipids in a given leaflet and the surface of this leaflet is shown in Figure 6A. Note that the surface area was determined at the level of the lipid polar head (choline). In the concave membrane region (inner tube leaflet), the area per lipid of mono and polyunsaturated PC was very close, suggesting a similar ability of the two PC species to compress at the level of the polar head. In contrast, we observed a strong difference for the convex (outer) leaflet: polyunsaturated lipids occupied a $20 \%$ larger surface than monounsaturated lipids. We also compared these values to the standard values observed on flat membrane. The transition from flat to convex leaflet is accompanied by a $53 \%$ increase in the surface area of polyunsaturated phospholipids as compared to $36 \%$ for monounsaturated ones (Fig. 6A). This analysis suggests that polyunsaturated lipids have a better ability to swell in the convex leaflet than monounsaturated lipids.

Figure 6B shows how the sn2 acyl chain of PC in the convex (outer) leaflet distributes between three main conformations (straight, moderately kinked, and highly kinked). Compared to 18:0-18:1 PC, 18:0-22:6 PC showed a 5-6-fold increase in the highly kinked conformation at the expense of the straight one. Last, we analyzed the geometry of PC as a function of its acyl chain content and its distribution between the inner and outer leaflets of the tube. By translation and rotation transformations, we overlapped all PC molecules present in one leaflet such as to get a side projection of the lipid. This projection gives the overall shape of 18:0-22:6 PC or 18:0-18:1 $\mathrm{PC}$ in regions of positive or negative curvature (Fig. $6 \mathrm{C}$ ).

In the concave leaflet, the 2D projections of 18:0-22:6 PC or 18:0-18:1 PC were very similar, suggesting that the acyl chains explored a similar volume. In the convex leaflet, the 2D projections of 18:0-22:6 PC or 18:0-18:1 PC were different. The polar head region of 18:0-22:6 PC was partially masked by its acyl chains, whereas the polar head of 18:0-18:1 PC was more visible. Performing the same analysis on either the $s n 1$ or $s n 2$ chains shows that this effect arose from the ability of the $s n 2$ polyunsaturated acyl chain to form a collar around the polar head, whereas the neighboring sn1 saturated acyl chain was rather straight and sank deeper in the bilayer (Fig. 6D).

Overall, this geometrical analysis indicates that, when present in the convex face of the membrane deformation, polyunsaturated phospholipids adapt their shape to membrane curvature.

\section{Discussion}

Our simulations suggest that the transbilayer distribution of polyunsaturated lipids is decisive for their facilitating effect on membrane deformation. When present in the concave face, 
polyunsaturated phospholipids provide no advantage over monounsaturated ones. When present in the convex face, polyunsaturated phospholipids have a positive effect, which is as large as that observed when these lipids are present on the two leaflets. This clear-cut effect of the transbilayer distribution of polyunsaturated lipids is observed whatever the than in some differentiated membranes. Molecular analysis suggests that polyunsaturated phospholipids are well adapted to regions of positive curvature because their acyl chains tend to invade the space left vacant by the polar head group. Thus, our simulations suggest a rationale for the enrichment of polyunsaturated lipids in the cytosolic leaflet of the plasma

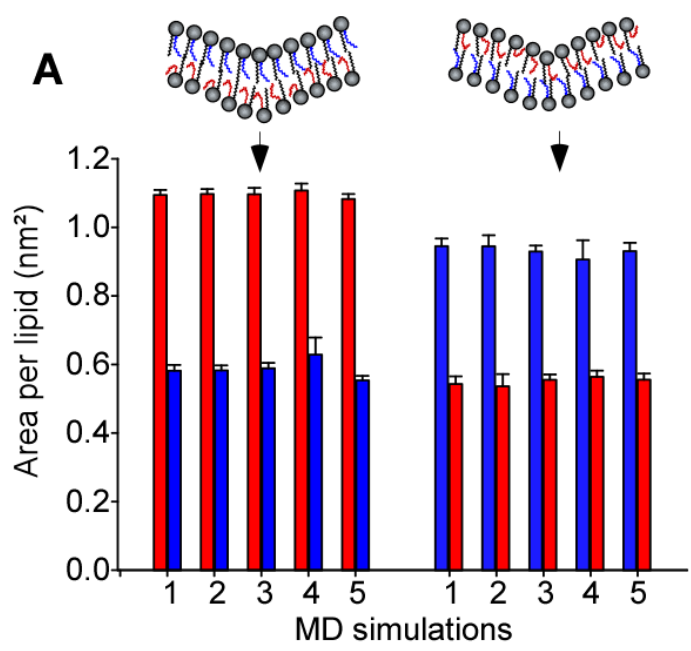

C

Overlay both acyl chains

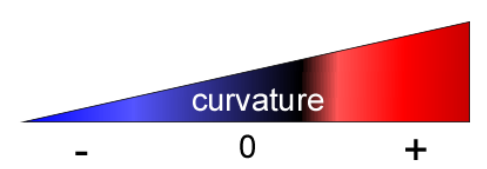

rirririr
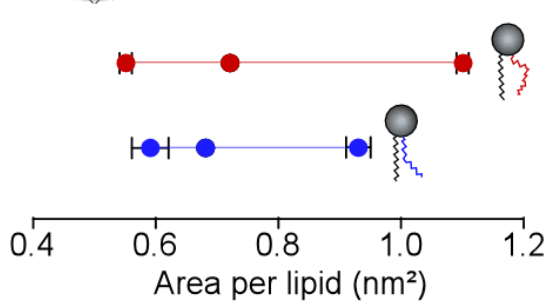

D Sn1
Overlay per acyl chain Sn2
B
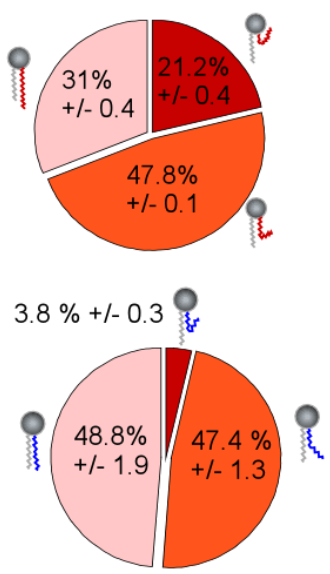
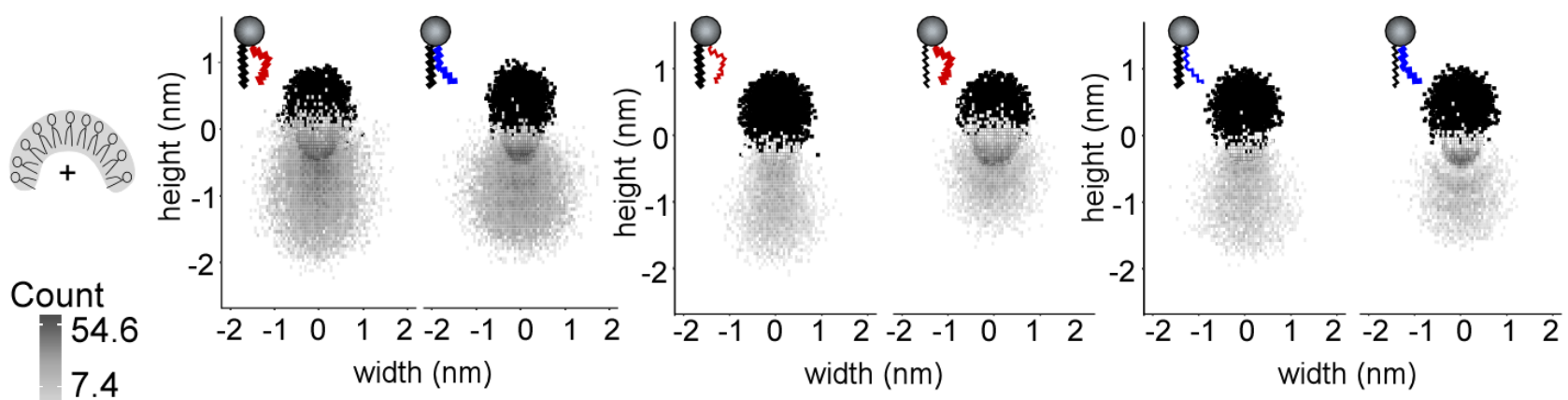

1
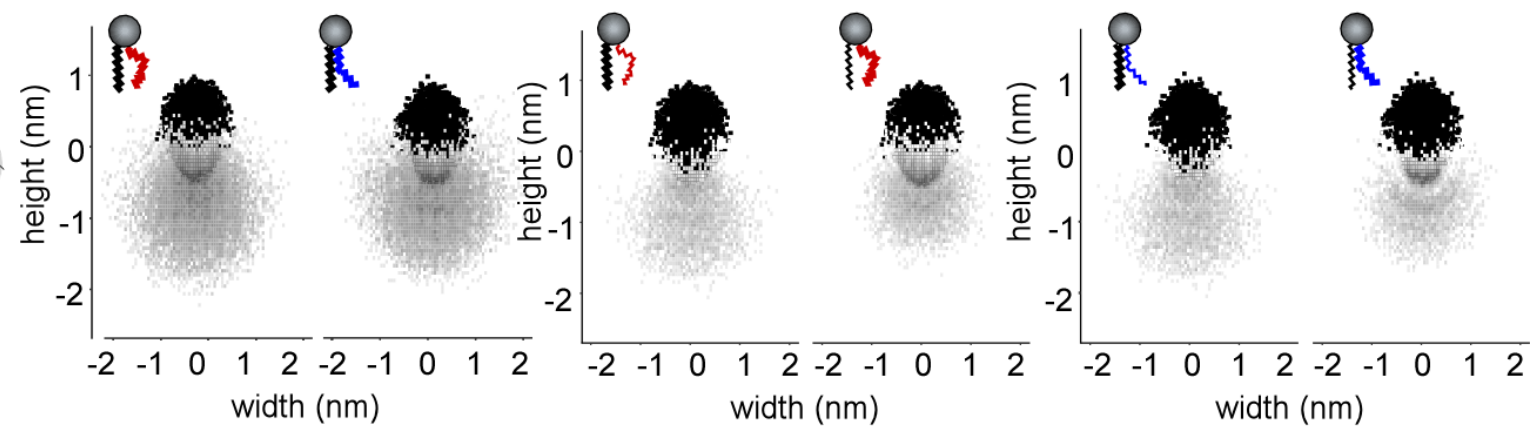

Fig 4 Conformation analysis. A. Area per lipid for each species in the outer and inner leaflets of the tube. The scheme represents the ability of each lipid to compress or dilate its area as a function of membrane geometry. B. Conformation distribution of mono or polyunsaturated PC when present in a convex membrane region. C, D. Overlay of the side projection of the indicated PC species according to the acyl chain profile and distribution in the outer (top) or inner leaflet of the tube. The polar head beads are shown in black, the acyl chain beads are shown in grey. The grey intensity reflects the number of events where a bin of the grid is occupied by a bead from the two acyl chains (C) or only from the indicated acyl chain

lipid class (PC, PE or PS) and occurs at concentrations similar membrane ${ }^{16}$ :

to

make 

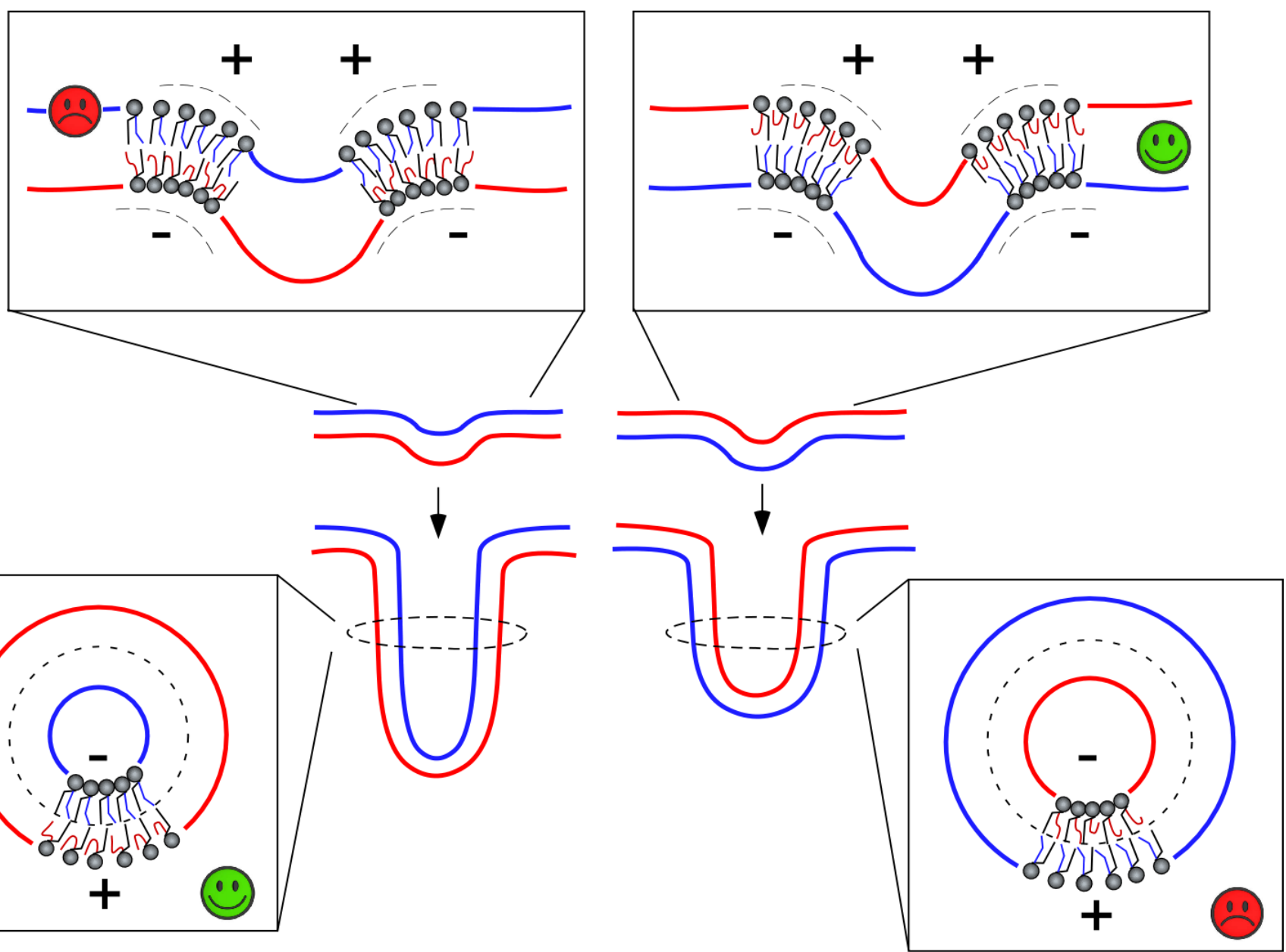

membrane well adapted to fast inward deformation, such as endocytosis.

Membrane deformation necessarily constrains the geometry of phospholipids ${ }^{22}$. The elementary volume available for a lipid in a convex leaflet has the shape of an inverted cone, whereas the elementary volume available for a lipid in a concave leaflet has a conical shape. One possibility to resolve these constraints is lipid sorting to match these elementary volumes

and lipid shape: a lipid with a conical shape might preferentially reside in the concave leaflet, whereas a lipid with an opposite shape might preferentially reside in the convex leaflet ${ }^{23}$. However, lipid sorting by curvature is readily counteracted by entropy as lipids diffuse rapidly and, in general, tend to $\mathrm{mix}^{24}$. Experimentally, lipid sorting by curvature is observed under stringent conditions such as extreme curvature or when the membrane is close to phase transition ${ }^{24-28}$.

Fig 5 Model of the impact of polyunsaturated lipids on membrane deformation as a function of their asymmetric distribution.

Polyunsaturated lipids offer an alternative mechanism for the adaptation of lipid composition to membrane shape. Instead of displaying an intrinsic shape, lipids with polyunsaturated acyl chains behave as contortionists: they adapt their conformation to membrane curvature ${ }^{7,29,30}$. The present study suggests that this adaptation occurs mostly in the convex leaflet. The analysis of the area per lipid (Fig. 6A) suggests that, in the concave leaflet, the main constraint for lipid packing is the size of the polar head group not the volume occupied by the acyl chains. In contrast, phospholipids in the convex leaflet have to occupy a large surface area at the level of the polar head group. Geometrical analysis suggest that the various kinked conformations of polyunsaturated acyl chains allow them to populate the interfacial region. Interestingly, this effect could explain the unexpected enrichment of PE in the outer surface of membrane tubes as observed in simulations ${ }^{31}$. Protrusions of the sn2 polyunsaturated acyl chain toward the interfacial region could alleviate the interfacial stress arising from the small area occupied by the polar head of PE.

The distribution of concave and convex surfaces in a forming tube or in a bud is more complex than in a closed structure such as a vesicle. At the connection between the tube and the flat parental membrane, the bilayer exhibits a saddle curvature where the two principal curvatures are opposite in sign, i.e. positive and negative. This geometry might explain why the impact of polyunsaturated lipids is different between the onset of membrane deformation (when a dome is forming) 
and during tube extension. As depicted in Figure 7, there are constraints in lipid packing that are specific to the saddle geometry and which make polyunsaturated lipids in the leaflet opposite to the pulling force advantageous for dome formation. When a tube has formed, the saddle geometry at the base remains, but its contribution to the overall energy of the tube should become negligible compared to the constraints in the tubular part itself. Under these conditions, polyunsaturated lipids in the outer leaflet of the tube are advantageous for elongation.

In the future, testing the impact of the transbilayer distribution of polyunsaturated lipids on membrane mechanics will require preparing asymmetric membranes, which is notoriously difficult. We note, however, that new protocols have recently been published to form asymmetric lipid bilayers ${ }^{32-35}$.

\section{Conclusions}

Biological membranes are asymmetric not only for the main lipid classes, which are defined by the polar head regions, but also for the lipid species, which are defined by the acyl chain profile. Lipids with polyunsaturated acyl chains preferentially reside in the cytosolic leaflet ${ }^{16}$. Our coarse-grained pulling simulations suggest that this distribution is highly favorable for endocytosis, because polyunsaturated phospholipids are well adapted to positive curvature. The presence of polyunsaturated phospholipids in the cytosolic side of the neuronal plasma membrane might contribute to the exceptional speed at which this membrane undergoes endocytosis ${ }^{36}$.

\section{Conflicts of interest}

There are no conflicts to declare.

\section{Acknowledgements}

This work was supported by a grant from the Fondation pour la recherche médicale (Convention DEQ20180339156 Equipes FRM 2018), and was granted by the HPC resources of CINES under the allocations $A 0040707362$ and $A 0060707362$ made by $\mathrm{GENCl}$. Computations have also been done on the Mesocentre SIGAMM machine hosted by the Observatoire de la Côte d'Azur. Marion Tiberti is a recipient of PhD fellowship from the ministère de l'enseignement supérieur, de la recherche et de l'innovation. We thank Meng-Chen Tsai and Hélène Barelli for comments on the manuscript.

\section{Notes and references}

$¥$ Footnotes relating to the main text should appear here.
1 J. H. Hurley, E. Boura, L.-A. Carlson and B. Róźycki, Cell, 2010, $143,875-887$.

2 M. M. Manni, J. Derganc and A. Čopič, Bioessays, 2017, 39.

3 W. Rawicz, K. C. Olbrich, T. J. Mclntosh, D. Needham and E. Evans, Biophys. J., 2000, 79, 328-339.

4 S. Morlot, V. Galli, M. Klein, N. Chiaruttini, J. Manzi, F. Humbert, L. Dinis, M. Lenz, G. Cappello and A. Roux, Cell, 2012, 151, 619-629.

5 M. M. Manni, M. L. Tiberti, S. Pagnotta, H. Barelli, R. Gautier and B. Antonny, eLife, 2018, 7, 15114

6 M. Pinot, S. Vanni, S. Pagnotta, S. Lacas-Gervais, L.-A. Payet, T. Ferreira, R. Gautier, B. Goud, B. Antonny and H. Barelli, Science, 2014, 345, 693-697.

7 B. Antonny, S. Vanni, H. Shindou and T. Ferreira, Trends in Cell Biology, 2015, 25, 427-436.

8 T. Harayama and H. Riezman, Nature Reviews Molecular Cell Biology, 2018, 19, 281-296.

9 H. Yabuuchi and J. S. O'Brien, The Journal of Lipid Research, 1968, 9, 65-67.

10 S. Takamori, M. Holt, K. Stenius, E. A. Lemke, M. Grønborg, D. Riedel, H. Urlaub, S. Schenck, B. Brügger, P. Ringler, S. A. Müller, B. Rammner, F. Gräter, J. S. Hub, B. L. De Groot, G. Mieskes, Y. Moriyama, J. Klingauf, H. Grubmüller, J. Heuser, F. T. Wieland and R. Jahn, Cell, 2006, 127, 831-846.

11 T. Ben Gedalya, V. Loeb, E. Israeli, Y. Altschuler, D. J. Selkoe and R. Sharon, Traffic, 2009, 10, 218-234.

12 T. J. Lagny and P. Bassereau, Interface Focus, 2015, 5, 20150038.

13 S. Baoukina, S. J. Marrink and D. P. Tieleman, Biophys. J., 2012, 102, 1866-1871.

14 J. A. Op den Kamp, Annu. Rev. Biochem., 1979, 48, 47-71.

15 A. J. Verkleij, R. F. Zwaal, B. Roelofsen, P. Comfurius, D. Kastelijn and L. L. van Deenen, Biochim. Biophys. Acta, 1973, 323, 178-193.

16 J. H. Lorent, L. Ganesan, G. Rivera-Longsworth, E. Sezgin, K. R. Levental, E. Lyman and I. Levental, bioRxiv, 2019, 698837.

17 B. Hess, C. Kutzner, D. van der Spoel and E. Lindahl, J Chem Theory Comput, 2008, 4, 435-447.

18 H. J. C. Berendsen, J. P. M. Postma, W. F. van Gunsteren, A. DiNola and J. R. Haak, The Journal of Chemical Physics, 1984, 81, 3684-3690.

19 H. I. Ingólfsson, T. S. Carpenter, H. Bhatia, P.-T. Bremer, S. J. Marrink and F. C. Lightstone, Biophys. J., 2017, 113, 2271-2280.

20 T. Harayama, M. Eto, H. Shindou, Y. Kita, E. Otsubo, D. Hishikawa, S. Ishii, K. Sakimura, M. Mishina and T. Shimizu, Cell Metabolism, 2014, 20, 295-305.

21 T. Hirama, S. M. Lu, J. G. Kay, M. Maekawa, M. M. Kozlov, S. Grinstein and G. D. Fairn, Nature Communications, 2017, 8, 1393.

22 C. Huang and J. T. Mason, Proceedings of the National Academy of Sciences, 1978, 75, 308-310.

23 L. V. Chernomordik and M. M. Kozlov, Annu. Rev. Biochem., 2003, 72, 175-207.

24 M. M. Kamal, D. Mills, M. Grzybek and J. Howard, Proc. Natl. Acad. Sci. U.S.A., 2009, 106, 22245-22250.

25 A. Tian and T. Baumgart, Biophys. J., 2009, 96, 2676-2688.

26 J. Derganc, Phys Biol, 2007, 4, 317-324.

27 I. R. Cooke and M. Deserno, Biophysj, 2006, 91, 487-495.

28 B. Sorre, A. Callan-Jones, J.-B. Manneville, P. Nassoy, J.-F. Joanny, J. Prost, B. Goud and P. Bassereau, Proc. Natl. Acad. Sci. U.S.A., 2009, 106, 5622-5626.

29 H. J. Risselada and S. J. Marrink, Phys Chem Chem Phys, 2009, 11, 2056-2067.

$30 \mathrm{H}$. Barelli and B. Antonny, Nature, 2009, 458, 159-160.

31 S. Baoukina, H. I. Ingólfsson, S. J. Marrink and D. P. Tieleman, Adv. Theory Simul., 2018, 1, 1800034-4. 
32 M. Doktorova, F. A. Heberle, B. Eicher, R. F. Standaert, J. Katsaras, E. London, G. Pabst and D. Marquardt, Nat Protoc, 2018, 13, 2086-2101.

33 T. A. Enoki and G. W. Feigenson, Biophys. J., 2019, 117, 1037-1050.

34 P. Heo, S. Ramakrishnan, J. Coleman, J. E. Rothman, J. B. Fleury and F. Pincet, Small, 2019, 15, e1900725.
35 S. Pautot, B. J. Frisken and D. A. Weitz, Proceedings of the National Academy of Sciences, 2003, 100, 10718-10721.

36 S. Watanabe, Q. Liu, M. W. Davis, G. Hollopeter, N. Thomas, N. B. Jorgensen and E. M. Jorgensen, eLife, 2013, 2, e00723. 